\title{
SPECIAL POINTS IN COMPACT SPACES
}

\author{
MURRAY BELL
}

(Communicated by Franklin D. Tall)

\begin{abstract}
Given a collection $\mathscr{C}$, of cardinality $\kappa$, of subsets of a compact space $X$, we prove the existence of a point $x$ such that whenever $C \in \mathscr{C}$ and $X \in \bar{C}$, there exists a $G_{\lambda}$-set $Z$ with $\lambda<\kappa$ and $x \in Z \subset \bar{C}$. We investigate the case when $\mathscr{E}$ is the collection of all cozerosets of $X$ and also when $X$ is a dyadic space. We apply this result to homogeneous compact spaces. Another application is a characterization of $2^{\omega_{1}}$ among dyadic spaces.
\end{abstract}

\section{INTRODUCTION}

A space $X$ is homogeneous if for every $x, y \in X$, there exists an autohomeomorphism $h$ of $X$ such that $h(x)=y$. This paper is concerned with homogeneous compact spaces. For a cardinal $\kappa$ and a collection $\mathscr{C}$ of subsets of a space $X$, we define what we mean by a $\mathscr{C}_{\kappa}$-point of $X$ (see Definition 2.1). Our main results are the Point Theorem and the Dyadic Cozero Point Theorem of $\S 2$ which prove the existence of $\mathscr{C}_{\kappa}$-points in compact spaces. In $\S 3$ we apply the results of $\S 2$ to homogeneous spaces and get the Homogeneous Theorem and the Dyadic Cozero Homogeneous Theorem. This paper contains a number of examples illustrating the sharpness of the theorems and also answering two questions previously posed by the author. Finally, we characterize $2^{\omega_{1}}$, among dyadic spaces, as the unique zero-dimensional, homogeneous compact space of weight $\omega_{1}$. This answers a question posed by B. Efimov for the case $\kappa=\omega_{1}$ of $2^{\kappa}$. We first proved this result assuming the continuum hypothesis $C H$. Subsequently, L. Shapiro removed this extra assumption. We show how it follows from our general theory.

Cardinals are initial ordinals, and $\omega$ and $\omega_{1}$ are the first two infinite cardinals. The cofinality of the cardinal $\kappa$ is denoted by $\operatorname{cf}(\kappa)$. The set of all subsets of $X$ is denoted by $\mathscr{P}(X)$. We use standard set-theory notions as in Kunen's Set Theory [Ku].

All spaces in this paper are assumed to be $T_{2}$, i.e., Hausdorff. We use standard topological notions as in Engleking's General Topology [En2] with one exception. We use the term cozeroset instead of the term functionally open. We emphasize two cardinal functions. The weight $w(X)$ of a space $X$ is the

Received by the editors February 1, 1993.

1991 Mathematics Subject Classification. Primary 54D30; Secondary 54F65, 54G20, 54C50.

Key words and phrases. Compact, cozeroset, dyadic, homogeneous, point.

Cheers to NSERC of Canada. 
least cardinal of a base for $X$, and the character $\chi(x, X)$ of $x$ in $X$ is the least cardinal of a local base at $x$ in $X$. We emphasize one topological property. A space $X$ is dyadic if $X$ is the continuous image of some Cantor Cube $2^{\kappa}$.

\section{THE POINT RESULTS}

We begin with the central definitions of our paper. Let $\kappa$ be an infinite cardinal. A subset $Z$ of a space $X$ is a $G_{\kappa}$-set (resp. $G_{<\kappa}$-set) if $Z=\bigcap_{\alpha<\lambda} U_{\alpha}$ where $\lambda \leq \kappa$ (resp. $\lambda<\kappa)$ and the $U_{\alpha}$ 's are open subsets of $X$. For $A \subset X$, we put $G_{\kappa}$-int $(A)=\bigcup\left\{Z \subset A: Z\right.$ is a $G_{\kappa}$-set of $\left.X\right\}$. We say that $A$ is $G_{\kappa^{-}}$ open in $X$ if $A=G_{\kappa}-\operatorname{int}(A)$. The preceding two notions yield two more if we uniformly replace $\kappa$ by $<\kappa$.

Definition 2.1. Let $\kappa$ be an infinite cardinal, and let $\mathscr{C} \subset \mathscr{P}(X)$. A point $x \in$ $X$ is a $\mathscr{C}_{\kappa}$-point of $X$ if whenever $C \in \mathscr{C}$ and $x \in \bar{C}$, then $x \in G_{<\kappa}$-int $(\bar{C})$.

Definition 2.2. Let $\kappa$ be an infinite cardinal. A point $x \in X$ is a $W_{\kappa}$-point of $X$ if there exists a disjoint clopen family $\mathscr{B}$ in $X$ of cardinality $\kappa$ such that every neighbourhood $N$ of $x$ intersects all but finitely many members of $\mathscr{B}$.

When the compact space $X$ is understood, we put $\mathscr{C O Z}=\{C \subset X: C$ is a cozeroset of $X\}$. Note that in a compact space $X$ a cozeroset is the same as an open $F_{\sigma}$ set.

Proposition 2.3. Let $X$ be a compact space, and let $\kappa$ be an infinite cardinal. Then, $a W_{\kappa}$-point is not a $\mathscr{C O} \mathscr{Z}_{\kappa}$-point.

Proof. Let $\mathscr{B}$ be a disjoint, clopen family of cardinality $\kappa$ witnessing the fact that $x$ is a $W_{\kappa}$-point of $X$. Let $\mathscr{C}$ be a countably infinite subfamily of $\mathscr{B}$. Then $C=\bigcup \mathscr{C}$ is a cozeroset of $X$ and $x \in \bar{C}$.

Let $\lambda<\kappa$, and let $Z$ be any $G_{\lambda}$-set with $x \in Z$. Using regularity, for each $\alpha<\lambda$, get a closed neighbourhood $N_{\alpha}$ of $x$ with $\bigcap_{\alpha<\lambda} N_{\alpha} \subset Z$. Using the $W_{\kappa}$-point property of $x$, choose $B \in \mathscr{B} \backslash \mathscr{C}$ such that for every finite $F \subset \lambda$, $B \cap \bigcap_{\alpha \in F} N_{\alpha} \neq \varnothing$. Using compactness of $X$, choose $p \in B \cap \bigcap_{\alpha<\lambda} N_{\alpha}$. Note that $B \cap C=\varnothing$. Thus, $p \in Z$ and $p \notin \bar{C}$. Hence $x$ cannot be a $\mathscr{C O O}_{\kappa}$-point of $X$.

Theorem 2.4 (Point Theorem). If $X$ is a compact space and $\mathscr{C}$ is a collection of subsets of $X$ with $|\mathscr{C}|=\kappa$, then there exists a $\mathscr{C}_{\kappa}$-point in $X$.

Proof. Enumerate $\mathscr{C}$ as $\left\{C_{\alpha}: \alpha<\kappa\right\}$. We will define, by induction on $\alpha<\kappa$, a sequence of zerosets $Z_{\alpha}$ of $X$. Put $Z_{0}=X$. At stage $\alpha$ if there exists a zeroset $Z$ such that $Z \cap \bigcap_{\beta<\alpha} Z_{\beta} \neq \varnothing$ and $Z \cap \bar{C}_{\alpha}=\varnothing$, then let $Z_{\alpha}$ be one such zeroset $Z$. If there does not exist such a zeroset $Z$, then put $Z_{\alpha}=X$.

At the end of this induction, by compactness, choose $x \in \bigcap_{\alpha<x} Z_{\alpha}$. Then $x$ is a $\mathscr{C}_{\kappa}$-point of $X$. To see this, let $C \in \mathscr{C}$ and assume that $x \in \bar{C}$. Choose $\alpha<\kappa$ with $C=C_{\alpha}$. We claim that $\bigcap_{\beta \leq \alpha} Z_{\beta} \subset \overline{C_{\alpha}}$. If not, then choose $y \in \bigcap_{\beta \leq \alpha} Z_{\beta} \backslash \overline{C_{\alpha}}$. Get a zeroset $Z$ with $y \in Z$ and $Z \cap \overline{C_{\alpha}}=\varnothing$. So at stage $\alpha$ we have that $Z_{\alpha} \cap \overline{C_{\alpha}}=\varnothing$. But this is a contradiction.

The Point Theorem only has content if each $x \in X$ has $\chi(x, X) \geq \kappa$, because a point of character $<\kappa$ is obviously a $\mathscr{C}_{\kappa}$-point. 
We are mostly interested when $\mathscr{C}=\mathscr{C O O Z}$. Since $|\mathscr{Q O Z Z}|=w(X)^{\omega}$ for compact spaces $X$, to avoid no content, we restate this special case as:

Theorem 2.5 (Cozero Point Theorem). If $X$ is compact and $w(X)=\kappa=\kappa^{\omega}$, then there exists a $\mathscr{C O O}_{\kappa}$-point in $X$.

We now give some examples to illustrate the sharpness, in general, of $\mathscr{C O O} \mathscr{Z}_{\kappa}$ in the Cozero Point Theorem. Obviously, if $\lambda<\kappa$, then having a $\mathscr{C O C Z}_{\lambda}$-point is a stronger result than having a $\mathscr{C O O} \mathscr{Z}_{\kappa}$-point. In certain spaces there are better results. Every point is a $\mathscr{C O} \mathscr{Z}_{\omega_{1}}$-point in any Cantor cube $2^{\kappa}$. Every point is a $\mathscr{C O Z} \mathscr{Z}_{\omega}$-point in any compact basically disconnected space. In the following we will use the basic fact that in a compact space $X$ a $W_{\kappa}$-point is not a $\mathscr{B} \mathscr{O} \mathscr{Z}_{\kappa}$ point.

Example 2.6. For every $\kappa>\omega$ there exists a compact $X_{\kappa}$ such that

- $w(X)=\kappa$;

- $X$ has no $\mathscr{C O O}_{\lambda}$-points, for $\lambda<\kappa$.

Proof. Case 1. $\kappa$ is a successor; $\kappa=\lambda^{+}$where $\lambda \geq \omega$. Put $\alpha \lambda=\lambda \cup\{\infty\}$, the Alexandroff one-point compactification of the discrete space $\lambda$. For $f \in(\alpha \lambda)^{\kappa}$, define $\operatorname{supp}(f)=\{\beta<\kappa: f(\beta) \neq \infty\}$. Set $X_{\kappa}=\left\{f \in(\alpha \lambda)^{\kappa}: f \mid \operatorname{supp}(f)\right.$ is a one-to-one function $\}$. As a subspace of $(\alpha \lambda)^{\kappa}, X_{\kappa}$ is closed and hence is compact. Let $f \in X_{\kappa}$. Choose $\beta \in \kappa \backslash \operatorname{supp}(f)$. For $\gamma<\lambda$, define $B_{\gamma}=\{g \in$ $\left.X_{\kappa}: g(\beta)=\gamma\right\}$. Then $\left\{B_{\gamma}: \gamma<\lambda\right\}$ witnesses the fact that $f$ is a $W_{\lambda}$-point. Hence, all points of $X_{\kappa}$ are $W_{\lambda}$-points, and therefore no point is a $\mathscr{Q O O}_{\lambda}$-point.

We mention that $X_{\omega_{2}}$ answers a question of the author raised in [Be1] about whether there exists a compact space in which every point is a $W_{\omega_{1}}$-point.

Case 2. $\kappa$ is a limit; $\kappa=\sum_{\alpha<\operatorname{cf}(\kappa)} \kappa_{\alpha}$ where for each $\alpha<\operatorname{cf}(\kappa), \kappa_{\alpha}$ is a successor cardinal $<\kappa$. Set $X_{\kappa}=\prod_{\alpha<\operatorname{cf}(\kappa)} X_{\kappa_{\alpha}}$ where the $X_{\kappa_{\alpha}}$ 's are as in Case 1. Fix $\lambda<\kappa$, and assume that $f \in X_{\kappa}$ is a $\mathscr{C O} \mathscr{Z}_{\lambda}$-point. Choose $\alpha<$ $\operatorname{cf}(\kappa)$ such that $\lambda<\kappa_{\alpha}<\kappa$. Then $f(\alpha)$ must be a $\mathscr{C O O} \mathscr{Z}_{\lambda}$-point of $X_{\kappa_{\alpha}}$-a contradiction.

The next example shows that the condition $\kappa=\kappa^{\omega}$ cannot be removed, in general, from the Cozero Point Theorem.

Example 2.7. There exists a compact space $Y$ of weight $\omega_{\omega}$ which has no $\mathscr{C O O}_{\omega_{\omega}}$-points.

Proof. For each $n<\omega$, define $Y_{n}=X_{\omega_{n+2}}$ as defined in Example 2.5 and put $Y=\prod_{n<\omega} Y_{n}$. For each $n<\omega$, let $\pi_{n}$ be the projection map onto $Y_{n}$.

Let $f \in Y$. For every $n<\omega, f(n)$ is not a $\mathscr{C O O} \mathscr{Z}_{\omega_{n+1}}$-point of $Y_{n}$ (by the previous example), so let $C_{n}$ be a cozeroset of $Y_{n}$ such that $f(n) \in$ $\overline{C_{n}} \backslash G_{\omega_{n}}-\operatorname{int}\left(\overline{C_{n}}\right)$. For every $n<\omega$, let $\left\langle C_{n}^{k}: k<\omega\right\rangle$ be an increasing sequence of cozerosets of $Y_{n}$ such that $C_{n}=\bigcup_{k<\omega} C_{n}^{k}$ and such that for every $k<\omega$, $\overline{C_{n}^{k}} \subset C_{n}$. For every $k<\omega$, define $D_{k}=\bigcap_{i \leq k} \pi_{k}^{-1}\left(C_{i}^{k}\right)$. Put $D=\bigcup_{k<\omega} D_{k}$. Then $D$ is a cozeroset of $Y$ and $f \in \bar{D}$.

Striving for a contradiction, assume that $f$ is a $\mathscr{C O O}_{\omega_{\omega}}$-point and choose $n<\omega$ and a closed $G_{\omega_{n}}$-set $Z$ with $f \in Z \subset \bar{D}$. For each $k<\omega$, choose a closed $G_{\omega_{n}}$-set $Z_{k}$ of $Y_{k}$ such that $f \in \prod_{k<\omega} Z_{k} \subset Z . Z_{n}$ cannot be 
contained in $\overline{C_{n}}$, so let $y \in Z_{n} \backslash \overline{C_{n}}$. Define $g \in Y$ by

$$
g(k)= \begin{cases}f(k) & \text { if } k \neq n, \\ y & \text { if } k=n .\end{cases}
$$

Then $g \in \prod_{k<\omega} Z_{k}$ and so $g \in \bar{D}$. But this is a contradiction as $\pi_{n}^{-1}\left(Y_{n} \backslash \overline{C_{n}}\right) \cap$ $\bigcap_{i<n} \pi_{i}^{-1}\left(Y_{i} \backslash C_{i}^{n}\right)$ is a neighbourhood of $g$ which is disjoint from $D=$ $\bigcup_{k<\omega}\left(\bigcap_{i \leq k} \pi_{k}^{-1}\left(C_{i}^{k}\right)\right)$.

It might be possible to replace the condition $\kappa=\kappa^{\omega}$ in the Cozero Point Theorem by the weaker condition $\operatorname{cf}(\kappa)>\omega$, but we do not know.

Question 2.8. If $X$ is a compact space with $w(X)=\kappa$ and $\operatorname{cf}(\kappa)>\omega$, then does $X$ have a $\mathscr{C O Z} \mathscr{Z}_{\kappa}$-point? (Of course, this is true if one assumes $G C H$; this question is particularly interesting for $\kappa=\omega_{1}$.)

We do have a positive answer to this question provided $X$ is a dyadic space.

Theorem 2.9 (Dyadic Cozero Point Theorem). If $X$ is a dyadic space and $w(X)=\kappa$ and $\operatorname{cf}(\kappa)>\omega$, then there exists $a \mathscr{B O O}_{\kappa}$-point in $X$.

Proof. Let $\phi: 2^{\kappa} \rightarrow X$. For each $\alpha<\kappa$, let $\pi_{\alpha}: 2^{\kappa} \rightarrow 2^{\alpha}$ be the projection map. We say that a subset $A$ of $2^{\kappa}$ depends on $\alpha$ if $A=\pi_{\alpha}^{-1} \pi_{\alpha}(A)$. We will define, by induction on $\alpha$, where $\omega \leq \alpha<\kappa$, a sequence of closed $G_{|\alpha|}$-sets $Z_{\alpha}$ of $X$. Put $Z_{\omega}=X$. At stage $\alpha$ put $W=\bigcap_{\beta<\alpha} Z_{\beta}$. Then $W$ is a closed $G_{|\alpha|}$-set. Put $Z_{\alpha}=$ some nonempty closed $G_{|\alpha|}$-set $Z \subset W$ such that if $Z^{\prime}$ is any nonempty closed $G_{|\alpha|}$-set with $Z^{\prime} \subset Z$, then $\pi_{\alpha}\left(\phi^{-1}\left(Z^{\prime}\right)\right)=\pi_{\alpha}\left(\phi^{-1}(Z)\right)$. Such a $Z$ exists since $2^{\alpha}$ has no strictly decreasing $|\alpha|^{+}$sequence of closed sets.

At the end of this induction, by compactness, choose $x \in \bigcap_{\alpha<x} Z_{\alpha}$. Then $x$ is a $\mathscr{C O O}_{\kappa}$-point in $X$. To see this, assume that $B \in \mathscr{C O Z}$ and that $x \in \bar{B}$. Then $\phi^{-1}(B)$ is a cozeroset in $2^{\kappa}$ and since $\operatorname{cf}(\kappa)>\omega$, we can choose an $\alpha<\kappa$ such that $\phi^{-1}(B)$ depends on $\alpha$. From $x \in Z_{\alpha} \cap \bar{B}$, it follows that $\phi^{-1}\left(Z_{\alpha}\right) \cap \overline{\phi^{-1}(B)} \neq \varnothing$.

We claim that $Z_{\alpha} \subset \bar{B}$. If not, then choose $y \in Z_{\alpha} \backslash \bar{B}$. Choose a zeroset $A \ni y$ such that $A \cap \bar{B}=\varnothing$. Put $Z^{\prime}=A \cap Z_{\alpha} . Z^{\prime}$ is a nonempty closed $G_{|\alpha|^{-}}$ set with $Z^{\prime} \subset Z_{\alpha}$. So at stage $\alpha$ we have that $\pi_{\alpha}\left(\phi^{-1}\left(Z^{\prime}\right)\right)=\pi_{\alpha}\left(\phi^{-1}\left(Z_{\alpha}\right)\right)$. $Z^{\prime} \cap \bar{B}=\varnothing$ implies that $\phi^{-1}\left(Z^{\prime}\right) \cap \overline{\phi^{-1}(B)}=\varnothing$. Since $\phi^{-1}(B)$ depends on $\alpha$, we get that $\overline{\phi^{-1}(B)}$ also depends on $\alpha$. So, $\pi_{\alpha}\left(\phi^{-1}\left(Z^{\prime}\right)\right) \cap \pi_{\alpha}\left(\overline{\phi^{-1}(B)}\right)=\varnothing$. Hence, $\pi_{\alpha}\left(\phi^{-1}\left(Z_{\alpha}\right)\right) \cap \pi_{\alpha}\left(\overline{\phi^{-1}(B)}\right)=\varnothing$. Thus, $\phi^{-1}\left(Z_{\alpha}\right) \cap \overline{\phi^{-1}(B)}=\varnothing-\mathrm{a}$ contradiction.

\section{APPLICATIONS TO HOMOGENEITY}

The first application is to resolve a question posed by the author in [Be2]. Let $X$ be a zero-dimensional compact space, and let $\mathscr{O} O$ be the family of clopen subsets of $X$. A family of sets $\mathscr{A}$ is centered if for every finite $\mathscr{F} \subset \mathscr{A}$ we have that $\bigcap \mathscr{F} \neq \varnothing$. Define $\operatorname{Cen}(X)=\left\{f \in 2^{\mathscr{B O}}:\{B \in \mathscr{B O}: f(B)=1\}\right.$ is a centered family\}. Cen $(X)$ is closed in $2^{8}$, hence it is compact. For each $B \in \mathscr{O}$, put $B^{*}=\{f \in \operatorname{Cen}(X): f(B)=1\}$. Then $B^{*}$ is a clopen subset of $\operatorname{Cen}(X)$. We consider $\operatorname{Cen}\left(\omega^{*}\right)$, where $\omega^{*}$ is the Stone-Cech remainder 
$\beta \omega \backslash \omega$. In the above paper we investigated $\operatorname{Cen}\left(\omega^{*}\right)$, showed that it in some sense the largest "Combinatorial" remainder of $\omega$, and mentioned that every point has $\pi$-character $c$. We asked whether $\operatorname{Cen}\left(\omega^{*}\right)$ was homogeneous.

Proposition 3.1. $\operatorname{Cen}\left(\omega^{*}\right)$ is not homogeneous.

Proof. Let $\overline{0}$ be the constant function 0 in $\operatorname{Cen}\left(\omega^{*}\right)$. Let $\mathscr{B}$ be a disjoint family of clopen sets in $\omega^{*}$ of cardinality $c$. The disjoint clopen family $\left\{B^{*}: B \in \mathscr{B}\right\}$ witnesses the fact that $\overline{0}$ is a $W_{c}$-point; thus, by Proposition 2.3, $\overline{0}$ is not a $\mathscr{O} \mathscr{Z}_{c}$-point. By the Cozero Point Theorem, since $w\left(\omega^{*}\right)=c=c^{\omega}$, $\operatorname{Cen}\left(\omega^{*}\right)$ must have a $\mathscr{C O Z}_{c}$-point. Since having a $\mathscr{C O O}_{c}$-point is a topological invariant, $\operatorname{Cen}\left(\omega^{*}\right)$ is not homogeneous and our proposition is proved.

It follows from the Point Theorem that $\omega^{*}$ has a $\mathscr{C O}_{c}$-point. An interesting question which we have not been able to resolve without assuming extra settheoretic assumptions is the following:

Question 3.2. Does $\omega^{*}$ have a point which is NOT a $\mathscr{C O Z}_{c}$-point?

Now, let us restate the Point Theorems for a homogeneous compact space. A family $\mathscr{C} \subset \mathscr{P}(X)$ is called invariant if for all $C \in \mathscr{C}$ and for all autohomeomorphisms $h$ of $X$ we have that $h(C) \in \mathscr{C}$. In this case the existence of one point gives us that all points are $\mathscr{C}_{\kappa}$-points.

Theorem 3.3 (Homogeneous Theorem). If $X$ is a homogeneous compact space and $\mathscr{C}$ is an invariant family with $|\mathscr{C}|=\kappa$, then for each $C \in \mathscr{C}, \bar{C}$ is $G_{<\kappa}$-open in $X$.

Theorem 3.4 (Cozero Homogeneous Theorem). If $X$ is a homogeneous compact space and $w(X)=\kappa=\kappa^{\omega}$ and $C \in \mathscr{O O Z}$, then $\bar{C}$ is $G_{<\kappa}$-open in $X$.

The Cozero Homogeneous Theorem cannot be improved by replacing $G_{<\kappa^{-}}$ open by a $G_{<x}$-set.

Example 3.5. There exists a homogeneous compact space $X$ such that

- $w(X)=c$;

- $X$ has a cozeroset $C$ such that $\bar{C}$ is not a $G_{<c}$-set.

Proof. Let $Y$ be the Alexandroff Double of the Cantor set $2^{\omega}$. Then $Y$ is a first countable, zero-dimensional, compact space with a dense set of isolated points. Put $X=Y^{\omega}$. By a result of Motorov [Mo], $X$ is homogeneous. Let $D$ be a countable, dense subset of $2^{\omega}$, and let $E$ be the isolated points of $Y$ which correspond to $D$. Then $E$ is a cozeroset of $Y$ and it is seen that $\bar{E}$ is not a $G_{<c}$-set in $Y$. Let $\pi$ be the projection map of $X$ onto the first factor space $Y$. It follows that if we put $C=\pi^{-1}(E)$, then $C$ is a cozeroset of $X$ and $\bar{C}$ is not a $G_{<c}$-set in $X$.

However, in a dyadic space, the Cozero Homogeneous Theorem can be improved to

Theorem 3.6 (Dyadic Cozero Homogeneous Theorem). If $X$ is a homogeneous dyadic space and $w(X)=\kappa^{+}$with $\kappa \geq \omega$ and $C \in \mathscr{C O O Z}$, then $\bar{C}$ is a $G_{\kappa}$-set. Proof. Applying homogeneity to the Dyadic Cozero Point Theorem we immediately get that if $C \in \mathscr{B O Z}$, then $\bar{C}$ is $G_{\kappa}$-open in $X$. In dyadic spaces, closed $G_{\kappa}$-open sets are actually $G_{\kappa}$-sets, so our result follows. A justification of this 
just-quoted dyadic fact goes as follows: Theorem 6 of Engelking [En1, p. 293] implies that in a Cantor cube, a closed $G_{\kappa}$-open set is actually a $G_{\kappa}$-set. The general dyadic case follows from an easy argument showing that this property is preserved under closed continuous images.

Efimov [Ef] asked whether there existed a zero-dimensional, homogeneous dyadic space not homeomorphic to $2^{\kappa}$. This was answered by Pashenkov [Pa], who showed that they do exist. However, the smallest weight example that he produced was of weight $2^{\omega_{1}}$. Using the Cozero Homogeneous Theorem plus the additional assumption of the Continuum Hypothesis, we showed that there could be no such example of weight $\omega_{1}$. Subsequently, L. Shapiro [Sh] removed the $\mathrm{CH}$ assumption completely from this result. After being informed of this, we proved the Dyadic Cozero Homogeneous Theorem. The reader is encouraged to read [Sh] for an inverse limit approach to this problem.

Corollary 3.7. The Cantor cube $2^{\omega_{1}}$ is topologically the only zero-dimensional, homogeneous dyadic space of weight $\omega_{1}$.

Proof. Let $X$ have all the properties in the corollary. Since $\chi(X)=w(X)$ in dyadic spaces and $X$ is homogeneous, we get that $X$ has uniform character $\omega_{1}$. Since, in a dyadic space, a regular closed set is the closure of some cozeroset, by applying the preceding theorem, we get that every regular closed set is a $G_{\omega}$-set.

To complete the proof, we now use the following result of Schepin [Sc]: A zero-dimensional dyadic space of uniform character $\omega_{1}$ in which every regular closed set is a $G_{\omega}$-set is homeomorphic to $2^{\omega_{1}}$. As this result does not explicitly appear in this reference, to help our readers, we explain it using notions and theorems from that paper. Theorem 18 on p. 10 implies that $X$ is opengenerated. Since $w(X)=\omega_{1}$, Theorem 3 on p. 2 implies that $X$ is $\operatorname{AE}(0)$. Finally, Theorem 1 on page 1 implies that $X$ is a Cantor cube.

\section{REFERENCES}

[Be1] M. Bell, Nonhomogeneity of powers of Cor images, Rocky Mountain J. Math. 22 (1992), 805-812.

[Be2] - Generalized dyadic spaces, Fund. Math. 125 (1985), 47-58.

[Ef] B. Efimov, Dyadic bicompacta, Trans. Moscow Math. Soc. 14 (1965), 229-267.

[En1] R. Engelking, Cartesian products and dyadic spaces, Fund. Math. 57 (1965), 287-303.

[En2] _ General topology, Sigma Ser. Pure Math., vol. 6, Heldermann Verlag, Berlin, 1989.

[Ku] K. Kunen, Set theory, Stud. Logic Found. Math., vol. 120, North-Holland, Amsterdam, 1980.

[Mo] D. Motorov, Zero-dimensional and linearly ordered bicompacta, Russian Math. Surveys 44 (1989), 190-191.

[Pa] V. V. Pashenkov, Extensions of compact spaces, Soviet Math. Dokl. 15 (1974), 43-47.

[Sc] E. Schepin, Functors and uncountable powers of compacta, Russian Math. Surveys 36 (1981), $1-71$.

[Sh] L. Shapiro, On homogeneities of dyadic bicompacta, manuscript, 1993.

Department of Mathematics, University of Manitoba, Winnipeg, Manitoba, Canada R3T 2N2

E-mail address: mbell@cc.umanitoba.ca 